\title{
Wiener's type regularity criteria on the complex plane
}

\author{
by JózeF Siciak (Kraków)
}

\begin{abstract}
We present a number of Wiener's type necessary and sufficient conditions (in terms of divergence of integrals or series involving a condenser capacity) for a compact set $E \subset \mathbb{C}$ to be regular with respect to the Dirichlet problem. The same capacity is used to give a simple proof of the following known theorem $[2,6]$ : If $E$ is a compact subset of $\mathbb{C}$ such that $d\left(t^{-1} E \cap\{|z-a| \leq 1\}\right) \geq$ const $>0$ for $0<t \leq 1$ and $a \in E$, where $d(F)$ is the logarithmic capacity of $F$, then the Green function of $\overline{\mathbb{C}} \backslash E$ with pole at infinity is Hölder continuous.
\end{abstract}

Introduction. Let $r$ and $R$ be real numbers with $1<r<R-2$. Given a subset $E$ of the disk $B \equiv B(a, R):=\{|z-a|<R\}$, let $h(z) \equiv h(z, E, B)$ be defined by the formula $h(z):=\sup \{u(z): u$ is a subharmonic function in $B$ such that $u \leq 0$ on $E$ and $u<1$ in $B\}$.

Then $h$ is the unique subharmonic function in $B$ such that: $0 \leq h \leq 1$ in $B ; h$ is harmonic in $B \backslash E ; h=0$ quasi-almost everywhere on $E$; and $\lim _{z \rightarrow \zeta} h(z)=1$ if $|\zeta-a|=R$.

One can check (see e.g. [10]) that the set function

$$
c(E) \equiv c(E ; B(a, R), \bar{B}(a, r)):=1-\sup _{|z-a|=r} h(z, E, B), \quad E \subset B,
$$

is a Choquet capacity with the property that a subset $E$ of $B$ is polar with respect to subharmonic functions iff $c(E)=0$. The set function $c(E)$ is called a capacity of the condenser $(E, B(a, R))$ with respect to the disk $\bar{B}(a, r)$, or condenser capacity of $E$ with respect to the disks $B(a, R)$ and $\bar{B}(a, r)$.

In the sequel $E$ denotes a polynomially convex compact subset of $\mathbb{C}$. Given a point $a$ of $E$, we define

$$
(*) \quad c(a, t):=c\left(a+t^{-1}(E \cap \bar{B}(a, t)-a)\right), \quad d(a, t):=d(E \cap \bar{B}(a, t))
$$

1991 Mathematics Subject Classification: 31A05, 31A15, 31A25.

Key words and phrases: subharmonic functions, logarithmic potential theory, Green function, regular points, Hölder Continuity Property.

Research supported by KBN Grant No 2 PO3A 05708. 
for all $t$ with $0 \leq t \leq 1$, where $d(F)$ denotes the transfinite diameter (logarithmic capacity) of a compact set $F$. Observe that $a+t^{-1}(E \cap \bar{B}(a, t)-a)$ is the portion of $E$ contained in $\bar{B}(a, t)$ scaled $t^{-1}$ times.

If $d(E)>0$, let $V_{E}$ denote the Green function of the unbounded component of $\mathbb{C} \backslash E$ with pole at $\infty$ (we put $V_{E}=0$ in the bounded components of $\mathbb{C} \backslash E)$.

Let $m$ and $\varrho_{n}$ be real numbers such that $m \geq 1$ and $0<\varrho_{n+1}<\varrho_{n}<$ $\varrho_{0}=1(n \geq 1)$. Put

$$
\delta\left(a, \varrho_{n}\right):=d\left(E \cap\left\{\varrho_{n+1} \leq|z-a| \leq \varrho_{n}\right\}\right) .
$$

The aim of this paper is to prove the following theorems:

I. $V_{E}$ is continuous at a iff

iff

$$
I:=\int_{0}^{1} \frac{d t}{t \log \frac{1}{d(a, t)}}=\infty
$$

iff

$$
J:=\int_{0}^{1} \frac{d t}{t \log \frac{m t}{d(a, t)}}=\infty
$$

$$
\mathcal{K}:=\int_{0}^{1} \frac{c(a, t)}{t} d t=\infty .
$$

II. If $1<A \leq \varrho_{n} / \varrho_{n+1} \leq B<\infty(n \geq 1)$ then $V_{E}$ is continuous at a iff

iff

$$
S_{4}:=\sum_{n=1}^{\infty} \frac{1}{\log \frac{1}{d\left(a, \varrho_{n}\right)}}=\infty
$$

iff

$$
S_{5}:=\sum_{n=1}^{\infty} \frac{1}{\log \frac{m \varrho_{n}}{d\left(a, \varrho_{n}\right)}}=\infty
$$

$$
S_{6}:=\sum_{n=1}^{\infty} c\left(a, \varrho_{n}\right)=\infty .
$$

III. If $1<A \leq\left(\log \varrho_{n+1}\right) /\left(\log \varrho_{n}\right) \leq B<\infty(n \geq 1)$ then $V_{E}$ is continuous at a iff

$$
S_{7}:=\sum_{n=1}^{\infty} \frac{\log \frac{1}{\varrho_{n}}}{\log \frac{1}{\delta\left(a, \varrho_{n}\right)}}=\infty
$$


iff

iff

$$
S_{8}:=\sum_{n=1}^{\infty} \frac{\log \frac{1}{\varrho_{n}}}{\log \frac{1}{d\left(a, \varrho_{n}\right)}}=\infty
$$

iff

$$
S_{9}:=\sum_{n=1}^{\infty} \frac{\log \frac{1}{\varrho_{n}}}{\log \frac{m \varrho_{n}}{\delta\left(a, \varrho_{n}\right)}}=\infty
$$

iff

$$
S_{10}:=\sum_{n=1}^{\infty} \frac{\log \frac{1}{\varrho_{n}}}{\log \frac{m \varrho_{n}}{d\left(a, \varrho_{n}\right)}}=\infty
$$

$$
S_{11}:=\sum_{n=1}^{\infty} c\left(a, \varrho_{n}\right) \log \frac{1}{\varrho_{n}}=\infty .
$$

IV. If $d(a, t) \geq \alpha t$ (resp. $c(a, t) \geq \beta)(0 \leq t \leq 1)$, where $\alpha$ and $\beta$ are positive constants, then there exist positive constants $\kappa$ and $\mu$ such that

$$
V_{E}(z) \leq \kappa \delta^{\mu}, \quad|z-a| \leq \delta \leq 1,
$$

i.e. $V_{E}$ is Hölder continuous at a. Moreover, $\kappa$ (resp. $\mu$ ) depends only on $\alpha$ (resp. on $\beta$ ) and $E$ (but not on $a)$.

Let

$$
w(E):=\frac{1}{\log \frac{1}{d(E)}}
$$

denote the Wiener capacity of $E \subset B(a, R)$. Then the integral $J$ can be written in the form $J=\int_{0}^{1} w(a, t) \frac{d t}{t}$, where $w(a, t):=w\left(a+(m t)^{-1}(E \cap\right.$ $\bar{B}(a, t)-a))$ is the Wiener capacity of the portion $E \cap \bar{B}(a, t)$ of $E$ scaled $(m t)^{-1}$ times.

The divergence of the integral $I$ (and of each of the series $S_{4}, S_{7}$ or $S_{8}$ ) is Wiener's well known necessary and sufficient condition for $E$ to be regular at $a$ (see e.g. [5]). Our proof of Theorems I-IV is based on the following estimates involving the condenser capacity $c$ defined by $(*)$ :

$$
\frac{\log \frac{R+1}{r-1}}{\log \frac{t(R+1)}{d(a, t)}} \leq c(a, t) \leq \frac{\log \frac{R-1}{r+1}}{\log \frac{t(R-1)}{d(a, t)}}, \quad 0<t \leq 1,
$$


and

$$
\begin{array}{r}
h\left(z, E \cap \bar{B}\left(a, \varrho_{n}\right), B\left(a, \varrho_{n} R\right)\right) \leq e^{-c_{n}-\ldots-c_{n+k}}, \\
|z-a| \leq r \varrho_{n+k}, n \geq 1, k \geq 0,
\end{array}
$$

where $c_{n}:=c\left(a, \varrho_{n}\right)$.

It is well known that the regularity (resp. Hölder Continuity Property, briefly $\mathrm{HCP}$ ) plays a very important role in the theory of multivariate polynomial approximation of holomorphic (resp. $\mathcal{C}^{\infty}$ ) functions on compact subsets of $\mathbb{C}^{N}$ (see e.g. [18, 8]). Compact sets with HCP also appear in a natural way in complex dynamics [2]. Therefore it is desirable to characterize compact subsets of $\mathbb{C}^{N}$ (resp. of $\mathbb{R}^{N}$ ) with these properties. In the case of $N=1$ we have necessary and sufficient criteria for regularity expressed in terms of capacities. The condenser capacity, given by $(*)$, can also be defined for compact subsets of $\mathbb{C}^{N}(N \geq 1)$, and it permits giving a sufficient Wiener's type condition for a compact subset $E$ of $\mathbb{C}^{N}$ to be regular [13]. However, if $N \geq 2$, unfortunately we do not know whether the condition is necessary for local regularity.

So far we have no reasonable necessary condition (expressed in terms of a capacity) for the Hölder Continuity Property (even on the complex plane). However, the following theorem is true.

V. If $E$ is a Cantor set associated with a sequence $\left\{l_{n}\right\}$ such that $l_{n+1} \leq$ $\frac{1}{2} l_{n}$ (see [7] for the definition), and $\lim _{n \rightarrow \infty} l_{n+1} / l_{n}$ exists, then the following conditions are equivalent:

(i) $\inf _{n \geq 1} \sqrt[n]{l}_{n}>0$;

(ii) $\inf _{n \geq 1} l_{n+1} / l_{n}>0$;

(iii) $\exists_{c \geq 1} \forall_{\varepsilon \in[0,1]} \forall_{t \in E} \exists_{t^{\prime} \in E} \varepsilon / c \leq\left|t-t^{\prime}\right| \leq \varepsilon$;

(iv) $d(a, t) \geq \alpha t$ for $0 \leq t \leq 1$ and $a \in E$ with $\alpha=$ const $>0$;

(v) $E$ has $H C P$, i.e. $V_{E}(z) \leq \kappa \delta^{\mu}$ if $|z-a| \leq \delta \leq 1, a \in E, z \in \mathbb{C}$;

(vi) $E$ has the Markov Property, i.e. for some $M, \sigma>0$,

$$
\left\|p_{\nu}^{\prime}\right\|_{E} \leq M \nu^{\sigma}\left\|p_{\nu}\right\|_{E}, \quad \nu=0,1, \ldots,
$$

where $p_{\nu}$ is any polynomial of degree $\leq \nu$.

Indeed, if $\lim l_{n+1} / l_{n}$ exists then (i) $\Leftrightarrow($ ii). One can check that (ii) $\Rightarrow$ (iii) (see e.g. [12]). By Pommerenke [9], (iii) $\Leftrightarrow$ (iv) for every compact set $E$ in $\mathbb{C}$. The implication (iv) $\Rightarrow(\mathrm{v})$ follows from IV. The implication (v) $\Rightarrow(v i)$ is well known (it follows from Cauchy inequalities). Finally, the implication (vi) $\Rightarrow$ (i) is due to W. Pleśniak [7].

COROLlary. The classical triadic Cantor set has all the properties (i) $-($ vi). 
Let us add that V. Totik [14] has recently shown that (i) $\Leftrightarrow(\mathrm{v}) \Leftrightarrow(\mathrm{vi})$ for all Cantor sets associated with $\left\{l_{n}\right\}$ under the only assumption that $l_{n+1} \leq \frac{1}{2} l_{n}$.

I would like to thank Professor Ch. Pommerenke for having informed me about a simple proof of HCP for uniformly perfect sets communicated to him by José Fernandez (in his letter dated June 28, 1994). Our method of proof of the implication (iv) $\Rightarrow(v)$ was inspired by that of Fernandez. Fernandez's proof was based on the Lemma of [3]. Estimates (**) may be considered as a modified version of that Lemma.

\section{Preliminaries}

1.1. Let $\operatorname{SH}(\Omega)$ denote the set of all subharmonic functions in an open subset $\Omega$ of $\mathbb{C}$. We say that a property $\mathcal{P}$ holds q.a.e. (quasi-almost everywhere) on $E$ if there exist a subset $A$ of $E$ and $W \in \mathrm{SH}(\mathbb{C})$ such that $W=-\infty$ on $A$, and the property $\mathcal{P}$ holds at each point of $E \backslash A$.

1.2. Given a compact subset $E$ of $\mathbb{C}$, define

$$
\Phi_{E}(z):=\sup _{\nu \geq 1} \Phi_{\nu}^{1 / \nu}(z) \equiv \lim _{\nu \rightarrow \infty} \Phi_{\nu}^{1 / \nu}(z)
$$

for all $z \in \mathbb{C}$, where

$\Phi_{\nu}(z):=\sup \left\{|p(z)|: p\right.$ is a polynomial of degree $\leq \nu$ with $\left.\|p\|_{E} \leq 1\right\}$.

The following theorem is well known $[10,15]$ :

1.3. TheOREM. (i) $V_{E}(z) \equiv \log \Phi_{E}(z)$ for all $z \in \mathbb{C}$, where $V_{E}(z):=\sup \left\{u(z): u \in \mathrm{SH}(\mathbb{C}), u \leq 0\right.$ on $\left.E, \sup _{\zeta \in \mathbb{C}}\{u(\zeta)-\log (1+|\zeta|)\}<\infty\right\}$.

(ii) If $d(E)>0$ (where $d(E)$ is the transfinite diameter (logarithmic capacity) of $E)$, then $V_{E}^{*}$ is the unique function $u \in \mathrm{SH}(\mathbb{C})$ with the following properties:

(1) $u(z) \geq 0$ in $\mathbb{C}, u(z)=0$ q.a.e. on $E$;

(2) $u$ is harmonic in $\mathbb{C} \backslash E$;

(3) $\lim _{z \rightarrow \infty}[u(z)-\log |z|]=\log (1 / d(E))$.

(iii) If $d(E)>0$, then

$$
V_{E}^{*}(z)=\int \log \frac{|z-a|}{d(E)} d \mu(a), \quad z \in \mathbb{C},
$$

where $\mu$ is a positive Borel measure such that $\operatorname{supp} \mu \subset E$ and $\mu(E)=1$ ( $\mu$ is called the equilibrium measure of $E$ ).

1.4. We say that a compact set $E$ is regular at a point $a \in E$ (or $a$ is a regular point of $E$ ) if $V_{E}^{*}(a)=0$. It is clear that $E$ is regular at $a$ if and 
only if $\lim _{\delta \rightarrow 0} \omega_{E}(a, \delta)=0$, where

$$
\omega_{E}(a, \delta):=\sup _{|z-a| \leq \delta} V_{E}(z)
$$

is the modulus of continuity of $E$ at $a$. In other words, $E$ is regular at $a$ iff $V_{E}^{*}$ is continuous at $a$. In particular, if $D=D(E)$ is the unbounded component of $\mathbb{C} \backslash E$, then by the Bouligand criterion $D$ is regular at $a \in \partial D$ with respect to the classical Dirichlet problem if and only if $E$ is regular at $a$.

Put

$$
\omega_{E}(\delta):=\sup \left\{\omega_{E}(a, \delta): a \in E\right\} .
$$

We say that $E$ has the Hölder Continuity Property (HCP) if

$$
\omega_{E}(\delta) \leq \kappa \delta^{\mu}, \quad 0<\delta \leq 1,
$$

where $\kappa$ and $\mu$ are positive constants.

1.5. Let $F$ be a compact subset of the unit interval $[0,1]$. Let $a$ be a point of a compact subset $E$ of $\mathbb{C}$ such that

$$
\forall_{t \in F} \quad\{|z-a|=t\} \cap E \neq \emptyset .
$$

Then

$$
V_{E}(z) \leq V_{F}(-|z-a|), \quad z \in \mathbb{C} .
$$

(For the proof see e.g. Lemma 3.1 of [11].) Hence $\omega_{E}(a, \delta) \leq \omega_{F}(0, \delta)$. In particular, if $E$ is a compact subset of $\mathbb{C}$ such that for each component $S$ of $E, \operatorname{diam} S \geq 2 r=$ const $>0$, then for all $a \in E, \omega_{E}(a, \delta) \leq \kappa \delta^{1 / 2}, 0<\delta \leq 1$, where $\kappa:=\frac{2}{r}(1+\sqrt{1+r})$. Therefore $E$ has HCP with exponent $\mu=1 / 2$. In particular each nontrivial continuum has HCP with exponent $\mu=1 / 2$.

1.6. If $E$ is a subset of an open bounded set $\Omega$, we define the zero-one extremal function by the formula

$$
h(z, E, \Omega):=\sup \{u(z): u \in \operatorname{SH}(\Omega), u \leq 0 \text { on } E, u<1 \text { in } \Omega\}, \quad z \in \Omega .
$$

One can easily check (see e.g. [10]) that

(1) $h^{*}(z, E, \Omega) \equiv 1$ iff $E$ is polar (i.e. $W=-\infty$ on $E$ for some $W \in$ $\mathrm{SH}(\mathbb{C}))$

(2) $h^{*}(z, E, \Omega)=h(z, E, \Omega)$ in $\Omega \backslash E$ and $h$ is harmonic in $\Omega \backslash E$;

(3) $h^{*}(z, E, \Omega)=0$ q.a.e. on $E$;

(4) if $F$ is a fixed regular compact subset of $\Omega$ then the set function

$$
c(E) \equiv c(E ; \Omega, F):=1-\sup _{z \in F} h(z, E, \Omega), \quad E \subset \Omega,
$$

is a Choquet capacity such that $E \subset \Omega$ is polar with respect to subharmonic functions if and only if $c(E ; \Omega, F)=0$.

1.7. Lemma. If

$$
c(a, t):=c(E \cap \bar{B}(a, t) ; B(a, t R), \bar{B}(a, t r)), \quad 0 \leq t \leq 1,
$$


then

$$
\begin{gathered}
\frac{\log \frac{R-1}{r+1}}{\log \frac{t(R-1)}{d(a, t)}} \leq c(a, t) \quad \text { if } 0<r<R-2<\infty, \\
c(a, t) \leq \frac{\log \frac{R+1}{r-1}}{\log \frac{t(R+1)}{d(a, t)}} \quad \text { if } 1<r<R<\infty, 0<t \leq 1,
\end{gathered}
$$

and

(iii) $\quad c(a, t) \equiv c\left(a+t^{-1}(E \cap \bar{B}(a, t)-a) ; B(a, R), \bar{B}(a, r)\right)$.

Proof. Put

$$
m(a, t, \varrho):=\inf _{\partial B(a, t \varrho)} V_{E \cap \bar{B}(a, t)}, \quad M(a, t, \varrho):=\sup _{\bar{B}(a, t \varrho)} V_{E \cap \bar{B}(a, t)} .
$$

Then

$$
\frac{V_{E \cap \bar{B}(a, t)}(z)}{M(a, t, R)} \leq h(z, E \cap \bar{B}(a, t), B(a, t R)) \leq \frac{V_{E \cap \bar{B}(a, t)}^{*}(z)}{m(a, t, R)}
$$

for all $z \in B(a, t R)$. Hence

$$
\frac{m(a, t, r)}{M(a, t, R)} \leq 1-c(a, t) \leq \frac{M(a, t, r)}{m(a, t, R)}
$$

and consequently

$$
\frac{m(a, t, R)-M(a, t, r)}{m(a, t, R)} \leq c(a, t) \leq \frac{M(a, t, R)-m(a, t, r)}{M(a, t, R)} .
$$

From the integral representation of $V_{E \cap \bar{B}(a, t)}^{*}$ with respect to the equilibrium measure of $E \cap \bar{B}(a, t)$ (see (iii) of Theorem 1.3(iii)) one gets the following inequalities:

$$
m(a, t, \varrho) \geq \log \frac{t(\varrho-1)}{d(a, t)}, \quad M(a, t, \varrho) \leq \log \frac{t(\varrho+1)}{d(a, t)}, \quad 1<\varrho \leq R,
$$

which imply inequalities (i) and (ii).

(iii) follows from the formula

$$
\begin{aligned}
h(a+t(z-a), E \cap \bar{B}(a, t), B(a, t R)) & \\
& \equiv h\left(z, a+t^{-1}(E \cap \bar{B}(a, t)-a), B(a, R)\right), \quad|z-a| \leq R,
\end{aligned}
$$

which is a direct consequence of the invariance of subharmonicity under complex linear transformations of coordinates.

1.8. Proposition. For a compact set $E \subset \mathbb{C}$ the following conditions are equivalent: 
(1) $E$ is locally regular at $a$, i.e. for all $\varrho>0, E(a, \varrho):=\widehat{E} \cap\{|z-a| \leq \varrho\}$ is regular at $a$, where $\widehat{E}$ denotes the polynomially convex hull of $E$;

(2) $E$ is regular at a;

(3) for every regular (with respect to the classical Dirichlet problem) open bounded set $\Omega$ containing $\widehat{E}$ one has $h^{*}(a, E, \Omega)=0$;

(4) there exists a regular open bounded set $\Omega$ such that $\widehat{E} \subset \Omega$ and $h^{*}(a, E, \Omega)=0$.

Proof. $\quad(1) \Rightarrow(2)$. It is sufficient to observe that $V_{E} \equiv V_{\hat{E}}$ and $V_{E} \leq$ $V_{E(a, \varrho)}$.

$(2) \Rightarrow(3)$ follows from the inequalities

$$
\frac{1}{M(E, \Omega)} V_{E}^{*}(z) \leq h^{*}(z, E, \Omega) \leq \frac{1}{m(E, \Omega)} V_{E}^{*}(z), \quad z \in \Omega,
$$

where $m(E, \Omega):=\inf _{\partial \Omega} V_{E}^{*}$ and $M(E, \Omega):=\sup _{\Omega} V_{E}^{*}$. In order to show $(*)$ recall that $V_{E^{\delta}} \uparrow V_{E}$ in $\mathbb{C}$ and $h\left(z, E^{\delta}, \omega\right) \uparrow h(z, E, \Omega)$ in $\Omega$ as $\delta \downarrow 0$, where $E^{\delta}:=\{z: \operatorname{dist}(z, E) \leq \delta\}$. Observe that $(*)$ is true for $E^{\delta}$ (by the maximum principle for harmonic functions in the open set $\left.\Omega \backslash E^{\delta}\right)$. Hence letting $\delta \downarrow 0$, we get $(*)$.

$(3) \Rightarrow(4)$ is obvious.

$(4) \Rightarrow(1)$. Without loss of generality we may assume that for every $r_{0}>0$ there is $r \in\left(0, r_{0}\right)$ such that $E \cap\{|z-a|=r\}=\emptyset$. Given $\varrho>0$ choose $r \in(0, \varrho)$ such that $E \cap\{|z-a|=r\}=\emptyset$ and $\bar{B}(a, r) \subset \Omega$. It is clear that $E(a, \varrho) \cap\{|z-a|=r\}=\emptyset$ and $d(E(a, \varrho))>0$ (otherwise $\left.h^{*}(a, E, \Omega)>0\right)$. Now by the maximum principle

$$
V_{E(a, \varrho)}^{*}(z) \leq M h^{*}(z, E, \Omega), \quad|z-a| \leq r,
$$

where

$$
M:=\sup _{|z-a|=r} V_{E(a, \varrho)}(z) / \inf _{|z-a|=r} h(z, E, \Omega) .
$$

Therefore $V_{E(a, \varrho)}^{*}(a)=0$.

1.9. Proposition. Let $\left\{\varrho_{n}\right\}$ be a sequence of real numbers such that $0<\varrho_{n+1}<\varrho_{n}<\varrho_{0}=1(n \geq 1)$ and $\lim _{n \rightarrow \infty} \varrho_{n}=0$. Let a be a fixed point of a compact set $E \subset \mathbb{C}$. Put

$$
\begin{aligned}
d(a, t) & :=d(E \cap\{|z-a| \leq t\}), \quad 0 \leq t \leq 1, \\
\delta\left(a, \varrho_{n}\right) & :=d\left(E \cap\left\{\varrho_{n+1} \leq|z-a| \leq \varrho_{n}\right\}\right)
\end{aligned}
$$

and

$$
I:=\int_{0}^{1} \frac{d t}{t \log \frac{1}{d(a, t)}} .
$$

Then the following statements are true: 
(1) We have

$$
\sum_{n=0}^{\infty} \frac{\log \frac{\varrho_{n}}{\varrho_{n+1}}}{\log \frac{1}{d\left(a, \varrho_{n+1}\right)}} \leq I=\sum_{n=0}^{\infty} \int_{\varrho_{n+1}}^{\varrho_{n}} \frac{d t}{t \log \frac{1}{d(a, t)}} \leq \sum_{n=0}^{\infty} \frac{\log \frac{\varrho_{n}}{\varrho_{n+1}}}{\log \frac{1}{d\left(a, \varrho_{n}\right)}}
$$

(2) If $1<A \leq \varrho_{n} / \varrho_{n+1}(n \geq 0)$, then

$$
\log A \sum_{n=1}^{\infty} \frac{1}{\log \frac{1}{d\left(a, \varrho_{n}\right)}} \leq I .
$$

(3) If $1<A \leq\left(\log \varrho_{n+1}\right) /\left(\log \varrho_{n}\right)(n \geq 0)$, then

$$
\left(1-\frac{1}{A}\right) \sum_{n=1}^{\infty} \frac{\log \frac{1}{\varrho_{n}}}{\log \frac{1}{d\left(a, \varrho_{n}\right)}} \leq I .
$$

(4) If $\varrho_{n} / \varrho_{n+1} \leq B<\infty(n \geq 0)$, then

$$
I \leq \log B \sum_{n=0}^{\infty} \frac{1}{\log \frac{1}{d\left(a, \varrho_{n}\right)}} .
$$

(5) If $\left(\log \varrho_{n+1}\right) /\left(\log \varrho_{n}\right) \leq B<\infty(n \geq 0)$ then

then

$$
I \leq \frac{\log \frac{1}{\varrho_{1}}}{\log \frac{1}{d(a, 1)}}+(B-1) \sum_{n=1}^{\infty} \frac{\log \frac{1}{\varrho_{n}}}{\log \frac{1}{d\left(a, \varrho_{n}\right)}} .
$$

(6) If $1<A \leq\left(\log \varrho_{n+1}\right) /\left(\log \varrho_{n}\right)(n \geq 1)$ and

$$
\sum_{n=1}^{\infty} \frac{\log \frac{1}{\varrho_{n}}}{\log \frac{1}{d\left(a, \varrho_{n}\right)}}=\infty
$$

$$
\sum_{n=1}^{\infty} \frac{\log \frac{1}{\varrho_{n}}}{\frac{1}{\delta\left(a, \varrho_{n}\right)}}=\infty
$$

(7) (Fundamental Inequality) If $0<r<R<\infty, R>1$ and $(*)$

$$
R \varrho_{n+1} \leq r \varrho_{n}, \quad n \geq 1,
$$


then

$$
h\left(z, E \cap \bar{B}\left(a, \varrho_{n}\right), B\left(a, \varrho_{n} R\right)\right) \leq e^{-c_{n}-\ldots-c_{n+k}}, \quad|z-a| \leq r \varrho_{n+k},
$$

for all $n \geq 1$ and $k \geq 0$, where $c_{n}:=c\left(a, \varrho_{n}\right)$.

P r o of. Statements (1)-(5) can be easily checked. To show (6) observe that by the subadditivity of the Wiener capacity $w(E):=1 / \log (1 / d(E))$ (see [5]) we have

$$
\frac{1}{\log \frac{1}{d\left(a, \varrho_{n}\right)}} \leq \frac{1}{\log \frac{1}{d\left(a, \varrho_{n+1}\right)}}+\frac{1}{\log \frac{1}{\delta\left(a, \varrho_{n}\right)}}, \quad n \geq k,
$$

where $k$ is so large that $\varrho_{n}<1 / 2$ for $n \geq k$. It follows from (S) that

$$
\frac{\log \frac{1}{\varrho_{k}}}{\log \frac{1}{d\left(a, \varrho_{k}\right)}}+\left(1-\frac{1}{A}\right) \sum_{n=k+1}^{\infty} \frac{\log \frac{1}{\varrho_{n}}}{\log \frac{1}{d\left(a, \varrho_{n}\right)}} \leq \sum_{n=k}^{\infty} \frac{\log \frac{1}{\varrho_{n}}}{\log \frac{1}{\delta\left(a, \varrho_{n}\right)}},
$$

which implies (6).

Now we prove (7). If $u$ is a subharmonic function in $B\left(a, \varrho_{n} R\right)$ such that $u \leq 0$ on $E \cap \bar{B}\left(a, \varrho_{n}\right)$ and $u \leq 1$ on $B\left(a, \varrho_{n} R\right)$, then $u(z) \leq 1-c_{n} \leq e^{-c_{n}}$ for all $z \in B\left(a, R \varrho_{n+1}\right)$, because $B\left(a, R \varrho_{n+1}\right) \subset B\left(a, r \varrho_{n}\right)$. Therefore

$$
u(z) \leq e^{-c_{n}} h\left(z, E \cap \bar{B}\left(a, \varrho_{n+1}\right), B\left(a, R \varrho_{n+1}\right)\right), \quad|z-a|<R \varrho_{n+1},
$$

as $E \cap \bar{B}\left(a, \varrho_{n+1}\right) \subset E \cap \bar{B}\left(a, \varrho_{n}\right)$. Since $u$ is arbitrary, we get

$$
h\left(z, E \cap \bar{B}\left(a, \varrho_{n}\right), B\left(a, R \varrho_{n}\right)\right) \leq e^{-c_{n}} h\left(z, E \cap \bar{B}\left(a, \varrho_{n+1}\right), B\left(a, R \varrho_{n+1}\right)\right)
$$

for all $z$ with $|z-a| \leq R \varrho_{n+1}$, which implies

$$
h\left(z, E \cap \bar{B}\left(a, \varrho_{n}\right), B\left(a, R \varrho_{n}\right)\right) \leq e^{-c_{n}-c_{n+1}}, \quad|z-a| \leq r \varrho_{n+1} .
$$

Repeating this procedure $k$ times, we get (§).

\section{Sufficient conditions}

2.1. Lemma. Let $0<r<R<\infty, R>1$, and let $\left\{\varrho_{n}\right\}$ be a sequence of positive numbers such that

$$
R \varrho_{n+1} \leq r \varrho_{n}, \quad n \geq 1 .
$$

If a is a point of a compact set $E$ in $\mathbb{C}$ such that

$$
\sum_{n=1}^{\infty} c\left(a, \varrho_{n}\right)=\infty
$$

then $E$ is regular at a. 
Proof. Given $n \geq 1$, choose $M$ so large that

$$
V_{E}(z) \leq M h\left(z, E \cap \bar{B}\left(a, \varrho_{n}\right), B\left(a, R \varrho_{n}\right)\right), \quad|z-a|<R \varrho_{n} .
$$

Given $\varepsilon>0$, by (\%) we can choose $k$ so large that

$$
M e^{-c_{n}-c_{n+1}-\ldots-c_{n+k}}<\varepsilon .
$$

Therefore by the Fundamental Inequality $(\S)$,

$$
V_{E}(z) \leq \varepsilon, \quad|z-a|<R \varrho_{n+k},
$$

which implies that $E$ is regular at $a$.

2.2. In the sequel $m, r$ and $R$ are real numbers with $m \geq 1$ and $1<r<$ $R-2$, and $\left\{\varrho_{n}\right\}$ denotes a sequence of real numbers such that

$$
0<\varrho_{n+1}<\varrho_{n}<\varrho_{0}=1(n \geq 1) \text { and } \lim _{n \rightarrow \infty} \varrho_{n}=0 .
$$

Given a compact set $E \subset \mathbb{C}$ and a point $a \in E$, we define

$$
\begin{aligned}
& I:=\int_{0}^{1} \frac{d t}{t \log \frac{1}{d(a, t)}}, \quad J:=\int_{0}^{1} \frac{d t}{t \log \frac{m t}{d(a, t)}}, \quad \mathcal{K}:=\int_{0}^{1} \frac{c(a, t)}{t} d t, \\
& S_{4}:=\sum_{n=0}^{\infty} \frac{1}{\log \frac{1}{d\left(a, \varrho_{n}\right)}}, \quad S_{5}:=\sum_{n=0}^{\infty} \frac{1}{\log \frac{m \varrho_{n}}{d\left(a, \varrho_{n}\right)}}, \quad S_{6}:=\sum_{n=0}^{\infty} c\left(a, \varrho_{n}\right), \\
& S_{7}:=\sum_{n=0}^{\infty} \frac{\log \frac{1}{\varrho_{n}}}{\log \frac{1}{\delta\left(a, \varrho_{n}\right)}}, \quad S_{8}:=\sum_{n=0}^{\infty} \frac{\log \frac{1}{\varrho_{n}}}{\log \frac{1}{d\left(a, \varrho_{n}\right)}}, \quad S_{9}:=\sum_{n=0}^{\infty} \frac{\log \frac{1}{\varrho_{n}}}{\log \frac{m \varrho_{n}}{d\left(a, \varrho_{n}\right)}}, \\
& S_{10}:=\sum_{n=0}^{\infty} \frac{\log \frac{1}{\varrho_{n}}}{\log \frac{m \varrho_{n}}{\delta\left(a, \varrho_{n}\right)}}, \quad S_{11}:=\sum_{n=0}^{\infty} c\left(a, \varrho_{n}\right) \log \frac{1}{\varrho_{n}} .
\end{aligned}
$$

2.3. TheOREM (Sufficient conditions). (i) If $I=\infty$ (or $\mathcal{K}=\infty$, or $J=\infty$ for some $m \geq 1$ ) then $E$ is regular at $a$.

(ii) If $1<A \leq \varrho_{n} / \varrho_{n+1}(n \geq 1)$ and $S_{4}=\infty$ (or $S_{6}=\infty$, or $S_{5}=\infty$ for some $m \geq 1$ ) then $E$ is regular at $a$.

(iii) If $1<A \leq\left(\log \varrho_{n+1}\right) /\left(\log \varrho_{n}\right)(n \geq 1)$ and $S_{7}=\infty$ (or $S_{8}=\infty$, or $S_{11}=\infty$, or $S_{10}=\infty$ for some $\left.m \geq 1\right)$ then $E$ is regular at $a$.

Pro of. (i) It is sufficient to show the following implications:

$$
\mathcal{K}=\infty \Rightarrow \exists_{m \geq 1} J=\infty \Rightarrow I=\infty \Rightarrow E \text { is regular at } a .
$$


The first implication follows from Lemma 1.7(ii) by putting $m=R+1$. In order to show the second implication fix $\lambda$ with $0<\lambda<1$ and put $\varrho_{n}:=\lambda^{2^{n}}(n \geq 1), \varrho_{0}=1$. Then

$$
\begin{aligned}
\infty & =J=\sum_{n=0}^{\infty} \int_{\varrho_{n+1}}^{\varrho_{n}} \frac{d t}{t \log \frac{m t}{d(a, t)}} \leq \sum_{n=0}^{\infty} \frac{\log \frac{\varrho_{n}}{\varrho_{n+1}}}{\log \frac{m \varrho_{n+1}}{d\left(a, \varrho_{n}\right)}} \\
& =\frac{\log \frac{1}{\varrho_{1}}}{\log \frac{m \varrho_{1}}{d(a, 1)}}+\sum_{n=1}^{\infty} \frac{\log \frac{1}{\varrho_{n}}}{\log \frac{m \varrho_{n+1}}{d\left(a, \varrho_{n}\right)}} \\
& =\frac{\log \frac{1}{\varrho_{1}}}{\log \frac{m \varrho_{1}}{d(a, 1)}}+\sum_{n=1}^{\infty} \frac{\log \frac{1}{\varrho_{n}}}{\log \frac{1}{d\left(a, \varrho_{n}\right)}} \varphi_{n}, \quad \text { where } \quad \varphi_{n}:=\frac{\log \frac{1}{d\left(a, \varrho_{n}\right)}}{\log \frac{m \varrho_{n+1}}{d\left(a, \varrho_{n}\right)}} .
\end{aligned}
$$

If $\sup _{n \geq 1} \varphi_{n}<\infty$, then $S_{8}=\infty$ and consequently by Proposition 1.9(3) we get $I=\infty$. If $\sup _{n \geq 1} \varphi_{n}=\infty$, then there exists a subsequence $\varrho_{n_{k}}$ such that $\left(\log \varrho_{n_{k+1}}\right) /\left(\log \varrho_{n_{k}}\right) \geq 2(k \geq 1)$ and $\lim _{k \rightarrow \infty} \varphi_{n_{k}}=\infty$. Observe that

$$
d\left(a, \varrho_{n_{k}}\right)=\left(m \varrho_{n_{k}+1}\right)^{\varphi_{n_{k}} /\left(\varphi_{n_{k}}-1\right)} \geq\left(m \varrho_{n_{k}+1}\right)^{2}=\left(m \varrho_{n_{k}}^{2}\right)^{2}, \quad k>k_{0} .
$$

Hence

$$
\frac{\log \frac{1}{\varrho_{n_{k}}}}{\log \frac{1}{d\left(a, \varrho_{n_{k}}\right)}} \geq \frac{1}{2\left[\frac{\log (1 / m)}{\log \left(1 / \varrho_{n_{k}}\right)}+2\right]} \geq \frac{1}{6}, \quad k>k_{1},
$$

which implies that $S_{8}=\infty$, and consequently $I=\infty$.

In order to show the last implication put $r=2, R=5, \varrho_{n}=(r / R)^{n}$ $(n \geq 1), \varrho_{0}=1$. By Lemma 1.7(i) we have

$$
\frac{\log \frac{4}{3}}{\log \frac{1}{d\left(a, \varrho_{n}\right)}} \leq \frac{\log \frac{4}{3}}{\log \frac{4 \varrho_{n}}{d\left(a, \varrho_{n}\right)}} \leq c\left(a, \varrho_{n}\right), \quad n>n_{0} .
$$

Hence by Proposition 1.9(4) we get $S_{6}=\infty$. Therefore by Lemma 2.1 the set $E$ is regular at $a$.

(ii) First let us prove the following implications:

$$
S_{4}=\infty \Rightarrow \forall_{m \geq 1} S_{5}=\infty \Rightarrow S_{6}=\infty \Rightarrow \exists_{m \geq 1} S_{5}=\infty .
$$

The first implication is obvious, the last one (resp. the second one) is a direct consequence of Lemma 1.7(ii) (resp. (i)). Now by (i) it remains to prove the 
implication

$$
\left(\varrho_{n} / \varrho_{n+1} \geq A>1(n \geq 1) \& S_{5}=\infty\right) \Rightarrow I=\infty .
$$

By Proposition 1.9(2),

$$
I \geq \log A \sum_{n=1}^{\infty} \frac{1}{\log \frac{1}{d\left(a, \varrho_{n}\right)}}=\log A \sum_{n=1}^{\infty} \frac{1}{\log \frac{m \varrho_{n}}{d\left(a, \varrho_{n}\right)}} \psi_{n}
$$

where

$$
\psi_{n}:=\frac{\log \frac{m \varrho_{n}}{d\left(a, \varrho_{n}\right)}}{\log \frac{1}{d\left(a, \varrho_{n}\right)}} .
$$

Consider two cases: $\varepsilon:=\inf _{n \geq 1} \psi_{n}>0$ and $\varepsilon=0$. In the first case we get $I \geq(\log A) S_{5} \varepsilon=\infty$, which implies that $I=\infty$. In the second case choose a subsequence $\left\{\varrho_{n_{k}}\right\}$ with $\left(\log \varrho_{n_{k+1}}\right) /\left(\log \varrho_{n_{k}}\right) \geq 2(k \geq 1)$ and $\lim _{k \rightarrow \infty} \psi_{n_{k}}=0$. Then

$$
d\left(a, \varrho_{n_{k}}\right)=\left(m \varrho_{n_{k}}\right)^{1 /\left(1-\psi_{n_{k}}\right)} \geq\left(m \varrho_{n_{k}}\right)^{2}, \quad k \geq k_{0},
$$

whence $S_{8}=\infty$, and consequently by Proposition 1.9(3), we get $I=\infty$.

(iii) First we check the following implications:

$$
S_{7}=\infty \Rightarrow \exists_{m \geq 1} S_{10}=\infty \Rightarrow \exists_{m \geq 1} S_{9}=\infty \Rightarrow S_{8}=\infty .
$$

The first two are obvious. In order to show the third, observe that

$$
\frac{\log \frac{1}{\varrho_{n}}}{\log \frac{m \varrho_{n}}{d\left(a, \varrho_{n}\right)}}=\frac{\log \frac{1}{\varrho_{n}}}{\log \frac{1}{d\left(a, \varrho_{n}\right)}} \varphi_{n}, \quad \text { where } \quad \varphi_{n}:=\frac{\log \frac{1}{d\left(a, \varrho_{n}\right)}}{\log \frac{m \varrho_{n}}{d\left(a, \varrho_{n}\right)}} .
$$

It is clear that if $\sup _{n \geq 1} \varphi_{n}<\infty$, then $S_{8}=\infty$. If $\lim _{k \rightarrow \infty} \varphi_{n_{k}}=\infty$, then

$$
d\left(a, \varrho_{n_{k}}\right)=\left(m \varrho_{n_{k}}\right)^{\varphi_{n_{k}} /\left(\varphi_{n_{k}}-1\right)} \geq\left(m \varrho_{n_{k}}\right)^{2}, \quad k>k_{0},
$$

which again implies that $S_{8}=\infty$.

By Lemma 1.7(ii),

$$
S_{11}=\infty \Rightarrow \exists_{m \geq 1} S_{9}=\infty .
$$

By Proposition 1.9(3) we get the implication

$$
\left(\frac{\log \varrho_{n+1}}{\log \varrho_{n}} \geq A>1(n \geq 1) \& S_{8}=\infty\right) \Rightarrow I=\infty .
$$

Statement (iii) now follows from $(\alpha),(\beta),(\gamma)$ and (i). The proof of Theorem 2.3 is complete. 
2.4. Corollary. If $\liminf _{t \downarrow 0} d(a, t) / t>0$, or $\liminf _{t \downarrow 0} c(a, t)>0$, or $\lim \sup _{t \downarrow 0} t^{-q} d(a, t)>0$ for some $q>0$, or $\limsup _{t \downarrow 0} c(a, t) \log (1 / t)>0$, then $E$ is regular at $a$.

In the first two cases, we get $I=\infty$ (resp. $\mathcal{K}=\infty$ ), so by (i), $E$ is regular at $a$. In the remaining two cases we can find $\varepsilon>0$ and $\left\{\varrho_{n}\right\}$ such that $\left(\log \varrho_{n+1}\right) /\left(\log \varrho_{n}\right) \geq 2(n \geq 1)$ and $\varrho_{n}^{-q} d\left(a, \varrho_{n}\right) \geq \varepsilon$ (resp. $\left.c\left(a, \varrho_{n}\right) \log \left(1 / \varrho_{n}\right) \geq \varepsilon\right)(n \geq 1)$. Hence $S_{8}=\infty$ (resp. $\left.S_{11}=\infty\right)$, which by (iii) implies the regularity of $E$ at $a$.

\section{Necessary conditions}

3.1. Lemma $[4,16]$. If a polynomially convex compact subset $E$ of $\mathbb{C}$ is regular at $a$ and

$$
\frac{\log \varrho_{n+1}}{\log \varrho_{n}} \leq B<\infty \quad(n \geq 1)
$$

then $S_{7}=\infty$.

Proof. Put $E_{n}:=E \cap\left\{\varrho_{n+1} \leq|z-a| \leq \varrho_{n}\right\}$ and $E^{n}:=E \cap\{|z-a|$ $\left.\leq \varrho_{n}\right\}$. By Proposition 1.8 it is enough to show that if the series $S_{7}$ is convergent then $E^{N}$ is not regular at $a$ for all $N$ sufficiently large. Put $\beta_{n}:=\sup _{D} V_{E_{n}}^{*}$, where $D:=\{|z-a|<1 / 2\}$. Fix $N \geq 1$ so large that $E^{N} \subset D$. The function

$$
u_{N}(z):=1+\sum_{n=N}^{\infty} \frac{V_{E_{n}}^{*}(z)-\beta_{n}}{\beta_{n}}
$$

is either subharmonic or identically $-\infty$ in $D$, because each term of the last series is a nonpositive subharmonic function in $D$. But it easily follows from Theorem 1.3(iii) that

$$
u_{N}(a) \geq 1-\sum_{n=N}^{\infty} \frac{\log \frac{1}{\varrho_{n+1}}}{\log \frac{1}{d\left(E_{n}\right)}} \geq 1-B \sum_{n=N}^{\infty} \frac{\log \frac{1}{\varrho_{n}}}{\log \frac{1}{d\left(E_{n}\right)}}>-\infty,
$$

as $V_{E_{n}}^{*}(a) \geq \log \left(\varrho_{n+1} / d\left(E_{n}\right)\right)$ and $\beta_{n} \leq \log \left(1 / d\left(E_{n}\right)\right)$. Therefore $u_{N}$ is subharmonic in $D$. Moreover, $u_{N}(z) \leq 0$ q.a.e. on $E_{n}$ for all $n \geq N$.

It is also clear that $u_{N}(z) \leq 1$ in $D$. Hence $u_{N}(z) \leq h^{*}\left(z, E^{N}, D\right)$ for all $z \in D$. In particular,

$$
h^{*}\left(a, E^{N}, D\right) \geq 1-\varepsilon_{N}, \quad \text { where } \quad \varepsilon_{N}:=B \sum_{n=N}^{\infty} \frac{\log \frac{1}{\varrho_{n}}}{\log \frac{1}{d\left(E_{n}\right)}},
$$


which implies that $h^{*}\left(a, E^{N}, D\right)>1 / 2$ if $N$ is sufficiently large. Therefore $E^{N}$ is not regular at $a$, and consequently $E$ is not regular at $a$.

3.2. Theorem (Necessary conditions). Let $E$ be a polynomially convex compact set regular at a. Then:

(I) $I=\infty, \mathcal{K}=\infty$, and $J=\infty$ for all $m \geq 1$.

(II) If $\varrho_{n} / \varrho_{n+1} \leq B<\infty(n \geq 1)$, then the series $S_{4}-S_{6}$ are divergent.

(III) If $\left(\log \varrho_{n+1}\right) /\left(\log \varrho_{n}\right) \leq B<\infty(n \geq 1)$ then the series $S_{7}-S_{11}$ are divergent.

Proof. (I) It is sufficient to show the following implications:

$E$ is regular at $a \Rightarrow I=\infty \Rightarrow \forall_{m \geq 1} J=\infty \Rightarrow \mathcal{K}=\infty$.

The first is a direct consequence of Lemma 3.1 and of Proposition 1.9(3). The second is obvious, and the third follows from Lemma 1.7(i).

(II) We know by (I) that $I=\infty$. Hence by Proposition 1.9(4) we get $S_{4}=\infty$. It is clear that $S_{4}=\infty \Rightarrow \forall_{m \geq 1} S_{5}=\infty$. Finally, the implication $S_{4}=\infty \Rightarrow S_{6}=\infty$ follows from Lemma 1.7(i).

(III) By Lemma 3.1 the series $S_{7}$ is divergent, which implies that so are $S_{8}$ and $S_{9}, S_{10}$ (for all $m \geq 1$ ). Finally, if $S_{8}$ is divergent then by Lemma 1.7(i) the series $S_{11}$ is divergent for all $m \geq 1$.

\section{Hölder Continuity Property}

4.1. Theorem (Capacity Scale Condition). Let $1 \leq r<R<\infty$ and let $\left\{\varrho_{n}\right\}$ be a sequence of real numbers such that $0<\varrho_{n}<1$ and

$$
\frac{R}{r} \leq \frac{\varrho_{n}}{\varrho_{n+1}} \leq B<\infty, \quad n \geq 1 .
$$

If $a$ is a point of a compact subset $E$ of $\mathbb{C}$ such that $c\left(a, \varrho_{n}\right) \geq m>0$, $(n \geq 1)$, then for every $\varrho>0$ the function $V_{E \cap \bar{B}(a, \varrho)}$ is Hölder continuous at a with exponent $\mu=m / \log B$ :

$$
V_{E \cap \bar{B}(a, \varrho)}(z) \leq M \delta^{m / \log B} \quad \text { if }|z-a| \leq \delta \leq 1,
$$

where $M=M(\varrho, r, R, m, B)$ depends only on $\varrho, r, R, m$ and $B$.

Pr o of. Given $\varrho>0$ take $n$ so large that $\varrho_{n} \leq \varrho$. By Proposition 1.9(7),

$$
h\left(z, E \cap \bar{B}\left(a, \varrho_{n}\right), B\left(a, R \varrho_{n}\right)\right) \leq e^{-m(k+1)}, \quad|z-a| \leq r \varrho_{n+k}, k \geq 1 .
$$

Given $\delta$ with $0<\delta \leq \min \left\{1, r \varrho_{n+1}\right\}$, choose $k$ such that $r \varrho_{n+k+1} \leq \delta \leq$ $r \varrho_{n+k}$. Then $B^{-k-1} r \varrho_{n} \leq \delta$ and consequently $-(k+1) \log B \leq \log \frac{\delta}{r \varrho_{n}}$, whence

$$
-m(k+1) \leq \log \left(\frac{\delta}{r \varrho_{n}}\right)^{m / \log B},
$$


which by (2) gives

$$
h\left(z, E \cap \bar{B}\left(a, \varrho_{n}\right), B\left(a, R \varrho_{n}\right)\right) \leq\left(\frac{1}{r \varrho_{n}}\right)^{m / \log B} \delta^{m / \log B}
$$

for all $z$ with $|z-a| \leq \delta \leq \min \left\{1, r \varrho_{n+1}\right\}$. There is $M_{1}=M_{1}(r, R, \varrho)>0$ such that

$$
V_{E \cap \bar{B}(a, \varrho)}(z) \leq M_{1} h\left(z, E \cap \bar{B}\left(a, \varrho_{n}\right), B\left(a, R \varrho_{n}\right)\right), \quad|z-a|<R \varrho_{n},
$$

which by (2a) gives the required result.

4.2. Corollary. (i) (Capacity Scale Condition) If $\inf _{0<t \leq 1} c(a, t)>0$, then $E$ has local HCP at a.

(ii) (Uniform Capacity Scale Condition I) If there exists a positive constant $m$ such that

$$
c(a, t) \geq m, \quad a \in E, 0<t \leq 1,
$$

then $E$ has HCP with exponent

$$
\mu=\frac{m}{\log \frac{R}{r}} .
$$

(iii) (Uniform Capacity Scale Condition II) Let $\left\{\varrho_{n}\right\}$ be a sequence satisfying (1). If there exists a positive constant $m$ such that

$$
c\left(a, \varrho_{n}\right) \geq m, \quad a \in E, n \geq 1,
$$

then $E$ has $H C P$ with exponent $\mu=m / \log B$.

(iv) (Uniform Logarithmic Capacity Scale Condition) If $E$ is uniformly perfect in the sense of Pommerenke [9], i.e.

$$
t^{-1} d(a, t) \equiv d\left(t^{-1} E \cap \bar{B}(a, t)\right) \geq m=\mathrm{const}>0, \quad 0 \leq t \leq 1, a \in E,
$$

then $E$ has $H C P$.

Observe that by Lemma 1.7 a compact set $E$ is uniformly perfect if and only if $c(a, t) \geq m>0$ for $a \in E$ and $0<t \leq 1$. We assume here that $1<r<R-2$.

4.3. R e m ark. The condition of (ii) (resp. (iii)) of Corollary 4.2 means that for each $t$ (resp. for each $n$ ) the portion of $E$ contained in the disk $\bar{B}(a, t)$ (resp. in the disk $\left.\bar{B}\left(a, \varrho_{n}\right)\right)$ scaled $1 / t$ times (resp. $1 / \varrho_{n}$ times) has the condenser capacity with respect to the balls $B(a, R)$ and $\bar{B}(a, r)$ (see Lemma 1.7(iii)) larger than a positive constant $m$. From the point of view of the condenser capacity a compact set satisfying the Uniform Capacity Scale Condition is "self-similar" at each of its points. Analogously, from the point of view of the transfinite diameter (equivalently: Wiener capacity) a compact set $E$ is uniformly perfect iff it is self-similar at each of its points. 
4.4. Given a compact set $E$ in $\mathbb{C}$, consider the following conditions:

(a) $E$ satisfies the Local Markov Inequality, i.e. for every $\nu \geq 1$ there exists $c_{\nu}$ such that

$$
\left\|p_{\nu}^{\prime}\right\|_{E \cap \bar{B}(a, \delta)} \leq \frac{c_{\nu}}{\delta}\left\|p_{\nu}\right\|_{E \cap \bar{B}(a, \delta)}, \quad a \in E, \quad 0<\delta \leq 1,
$$

where $p_{\nu}$ is any polynomial of degree $\leq \nu$;

(b) $E$ is uniformly perfect, i.e. $\exists_{c \geq 1} \forall_{\varepsilon \in(0,1]} \forall_{z \in E} \exists_{z^{\prime} \in E} \varepsilon / c \leq\left|z-z^{\prime}\right| \leq \varepsilon$;

(c) E satisfies the Uniform Logarithmic Capacity Scale Condition, i.e.

$$
d\left(t^{-1} E \cap\{|z-a| \leq t\}\right) \geq m=\text { const }>0, \quad a \in E, 0<t \leq 1 ;
$$

(d) $E$ has the Hölder Continuity Property, i.e. for some $\kappa, \mu>0$,

$$
V_{E}(z) \leq \kappa|z-a|^{\mu}, \quad a \in E, z \in \mathbb{C},|z-a| \leq 1 ;
$$

(e) $E$ satisfies the Markov Inequality, i.e. for some $M, \sigma>0$,

$$
\left\|p_{\nu}^{\prime}\right\|_{E} \leq M \nu^{\sigma}\left\|p_{\nu}\right\|_{E}, \quad \nu \geq 1 .
$$

It is known that

$$
(\mathrm{a}) \Leftrightarrow(\mathrm{b}) \Leftrightarrow(\mathrm{c}) \Rightarrow(\mathrm{d}) \Rightarrow(\mathrm{e})
$$

The equivalence $(\mathrm{a}) \Leftrightarrow(\mathrm{b})$ is due to Wallin and Wingren [17 ] (see also [6]). The equivalence $(\mathrm{b}) \Leftrightarrow(\mathrm{c})$ is due to Pommerenke [9]. As already observed in 4.2 the implication $(\mathrm{c}) \Rightarrow(\mathrm{d})$ follows from Theorem 4.1. Other proofs of this implication were earlier given by Lithner [6] and José Fernandez (in a letter dated June 28, 1994). The present author does not know who was the first to prove the implication (c) $\Rightarrow(\mathrm{d})$. In the book [2] (pages 64 and 138) this implication is stated without proof as if it were well known.

The last implication $(\mathrm{d}) \Rightarrow(\mathrm{e})$ is known since a long time; it follows from the Cauchy integral formula (or from the Cauchy inequalities).

As already mentioned in the introduction, for Cantor sets associated with a sequence $\left\{l_{n}\right\}$ such that $l_{n+1} \leq \frac{1}{2} l_{n}$ and the $\operatorname{limit}_{\lim } \lim _{n \rightarrow \infty} l_{n+1} / l_{n}$ exists, all the above conditions are equivalent.

Question. Which (if any) of the implications $(\mathrm{e}) \Rightarrow(\mathrm{d}),(\mathrm{e}) \Rightarrow(\mathrm{c})$, or $(\mathrm{d}) \Rightarrow(\mathrm{c})$ is true for all compact sets $E$ in $\mathbb{C}$ ?

4.5. EXAMPLE. Let $\left\{l_{n}\right\}$ be a sequence of positive real numbers with $l_{n+1} \leq \frac{1}{2} l_{n}(n \geq 0), l_{0}=1$. Put $F:=\{0\} \cup \bigcup_{n=0}^{\infty}\left[l_{n}-l_{n+1}, l_{n}\right]$.

(i) If $\sum_{n=0}^{\infty} \frac{1}{\log \left(1 / l_{n}\right)}=\infty\left(\right.$ or $\lim \sup _{n \rightarrow \infty} l_{n}^{-q} l_{n+1}>0$ for some $\left.q>0\right)$, then $F$ is regular.

(ii) If

$$
\inf _{n \geq 1} l_{n+1} / l_{n} \geq \alpha=\text { const }>0
$$


then

$$
d(a, t):=d(F \cap\{|z-a| \leq t\}) \geq \frac{\alpha^{2}}{8} t, \quad 0 \leq t \leq 1, a \in F .
$$

Pr o of. (i) follows from Theorem 2.3(ii) (resp. from Corollary 2.4).

(ii) First we shall show that

$$
d(0, t) \geq \frac{\alpha^{2}}{4} t, \quad 0 \leq t \leq 1 .
$$

Indeed, given $t$ with $0 \leq t \leq 1$, there exists $n$ such that $l_{n+1}<t \leq l_{n}$. Therefore

$$
d(0, t) \geq d\left(0, l_{n+1}\right) \geq \frac{1}{4} l_{n+2} \geq \frac{\alpha^{2}}{4} l_{n} \geq \frac{\alpha^{2}}{4} t .
$$

Given $a \in F$, there exists $n$ such that $a \in\left[l_{n}-l_{n+1}, l_{n}\right]$. It is clear that $d(a, t) \geq \frac{t}{4}$, when $0 \leq t \leq \frac{1}{2} l_{n+1}$. If $\frac{1}{2} l_{n+1}<t \leq l_{n}$, then $d(a, t) \geq$ $d\left(a, \frac{1}{2} l_{n+1}\right) \geq \frac{1}{8} l_{n+1} \geq \frac{\alpha}{8} l_{n} \geq \frac{\alpha}{8} t$. Finally, if $l_{n}<t \leq 1$, then $d(a, t) \geq$ $d(0, t)$, which by $(*)$ gives $d(a, t) \geq \frac{\alpha^{2}}{4} t$. It is clear that $\alpha \leq \frac{1}{2}$. Therefore $d(a, t) \geq \frac{\alpha^{2}}{8} t$ for $a \in F$ and $0<t \leq 1$.

\section{References}

[1] L. Białas and A. Volberg, Markov's property of the Cantor ternary set, Studia Math. 104 (1993), 259-268.

[2] L. Carleson and T. W. Gamelin, Complex Dynamics, Springer, 1993.

[3] W. Hayman and Ch. Pommereneke, On analytic functions of bounded mean oscillation, Bull. London Math. Soc. 10 (1978), 219-224.

[4] O. D. Kellogg and F. Vasilesco, A contribution to the theory of capacity, Amer. J. Math. 51 (1929), 515-526.

[5] N. S. Landkof, Foundations of Modern Potential Theory, Nauka, Moscow, 1966.

[6] J. Lithner, Comparing two versions of Markov's inequality on compact sets, J. Approx. Theory 77 (1994), 202-211.

[7] W. Pleśniak, A Cantor regular set which does not have Markov's property, Ann. Polon. Math. 51 (1990), 269-274.

[8] - Markov's inequality and the existence of an extension operator for $C^{\infty}$ functions, J. Approx. Theory 61 (1990), 106-117.

[9] Ch. Pommerenke, Uniformly perfect sets and the Poincaré metric, Arch. Math. (Basel) 32 (1980), 192-199.

[10] J. Siciak, Extremal Plurisubharmonic Functions and Capacities in $\mathbb{C}^{n}$, Sophia Kokyuroku in Math. 14, Sophia University, Tokyo, 1982.

[11] - Rapid polynomial approximation on compact sets in $\mathbb{C}^{N}$, Univ. Iagel. Acta Math. 30 (1993), 145-154.

[12] - Compact sets in $\mathbb{R}^{n}$ admitting polynomial inequalities, Trudy Mat. Inst. Steklov. 203 (1994), 441-448.

[13] - Wiener's type sufficient conditions in $\mathbb{C}^{N}$, to appear.

[14] V. Totik, Markoff constants for Cantor sets, to appear.

[15] M. Tsuji, Potential Theory in Modern Function Theory, Maruzen, Tokyo, 1959. 
[16] C. de la Vallée-Poussin, Points irréguliers. Détermination des masses par les potentiels, Bull. Cl. Sci. Bruxelles (5) 24 (1938), 672-689.

[17] H. Wallin and P. Wingren, Dimension and geometry of sets defined by polynomial inequalities, J. Approx. Theory 69 (1992), 231-249.

[18] J. L. Walsh, Interpolation and Approximation by Rational Functions in the Complex Domain, Amer. Math. Soc. Colloq. Publ. 20, Amer. Math. Soc., Providence, R.I., 1935. Third edition, 1960.

[19] N. Wiener, The Dirichlet problem, J. Math. Phys. Mass. Inst. Techn. 3 (1924), $127-146$

Institute of Mathematics

Jagiellonian University

Reymonta 4

30-059 Kraków, Poland

E-mail: siciak@im.uj.edu.pl 